\title{
Evaluation of spectral domain and time domain optical coherence tomography findings in toxoplasmic retinochoroiditis
}

This article was published in the following Dove Press journal:

Clinical Ophthalmology

16 May 201 I

Number of times this article has been viewed

\section{Bruno Diniz \\ Caio Regatieri \\ Rafael Andrade \\ Andre Maia}

Department of Ophthalmology, Federal University of São Paulo, São Paulo, Brazil
Correspondence: Bruno Diniz Rua T-62 n 1097 apt 1600 Setor Bueno, Goiania, GO, Brazil 74223-180

Tel +55 I I 99686303

Email drbrunodiniz@yahoo.com
Purpose: To investigate spectral domain optical coherence tomography (SD-OCT) findings and compare them with time domain (TD)-OCT imaging of macula and retinochoroiditis lesions of patients with toxoplasmosis.

Design: Prospective cross-sectional study.

Methods: Ten eyes of 10 patients with active toxoplasmic retinochoroiditis were included. Morphologic features from the macula and retinochoroiditis lesions were obtained at baseline and at 6-week follow up. Scan acquisition protocols for TD-OCT included raster and radial lines through the retinochoroiditis lesion, fast macular, and a linear scan from the lesion to the fovea, whereas the acquisition protocols for SD-OCT also included horizontal volume scans at the lesion site and at the macula. Thickness measurements obtained by SD-OCT were analyzed.

Results: At baseline, macular serous retinal detachment was observed in five patients; two of them only seen by SD-OCT. Retinochoroidal lesions were $4260 \mu \mathrm{m}$ distant from the fovea on average $(\mathrm{R}=681-7130)$ and this distance had an indirect correlation to the presence of macular detachment. Epiretinal membrane and vitreo-macular traction were also observed. The posterior hyaloid was not identified in four patients by TD-OCT and only in one by SD-OCT at baseline. Perilesional subretinal fluid was observed in two patients. The median retinal thickness significantly decreased at the retinochoroiditis lesion $(P=0.0004)$, and all the patients remained with disorganized retinal layers reflectivity at follow up.

Conclusion: SD-OCT is a useful tool in the diagnosis of macular changes related with toxoplasmic retinochoroiditis. SD-OCT is superior in evaluating retinal changes associated with ocular toxoplasmosis.

Keywords: toxoplasmosis, retinochoroiditis, optical coherence tomography

\section{Introduction}

Toxoplasmosis is the most common cause of intraocular inflammation and posterior uveitis in immunocompetent patients. ${ }^{1,2}$ It is caused by Toxoplasma gondii, which is an obligate intracellular parasite responsible for zoonotic infection in humans and other mammals. Ocular toxoplasmosis generally affects children and young people (25-45 years) and is characterized by recurrences that could ultimately lead to permanent visual loss.

Active lesions attack the inner retinal layers adjacent to an old chorioretinal scar and present as grey-white focus of retinal necrosis with adjacent choroiditis, vasculitis, hemorrhage, and vitreitis. ${ }^{3}$ Cicatrization occurs from the periphery towards the center of the lesion, with variable pigmentary changes. Anterior uveitis is a common finding, with mutton-fat keratic precipitates, cells and flare, and posterior synechiae. ${ }^{4}$ 
Optical coherence tomography (OCT) is now proven to be an effective noninvasive investigation in detecting pathologic features in uveitis and is gaining more and more popularity as an ancillary investigation. It can be used to assist the diagnosis and be repeated safely during follow-up to monitor response to any intervention. ${ }^{5}$

Recently, the introduction of spectral-domain (SD)-OCT has improved the image resolution, imaging speed, and sensitivity with improved resolution, volume rendering with three-dimensional imaging, and mapping of individual retinal layers. ${ }^{6}$ SD-OCT has the advantage of detecting small changes in the structure of the retinal layers and subretinal space, allowing precise anatomic detection of structural changes that may correspond to progression or regression of the toxoplasmic lesions. The practicability of using the additional information obtained by SD-OCT in clinical practice is not yet known when concerning toxoplasmic retinochoroiditis.

The present study investigated SD-OCT findings of macula and toxoplasmic retinochoroiditis lesions; and compared these findings with time domain (TD)-OCT imaging.

\section{Methods}

A total of 10 eyes from 10 patients consecutively diagnosed with active toxoplasmic retinochoroiditis in the Retina Service were enrolled in this prospective study. This study was approved by the Ethics and Research Review Board of Federal University of São Paulo (CEP-UNIFESP), and informed consent was obtained from participants in accordance with the tenets of the Declaration of Helsinki.

Patients with less than 5 days of symptoms and typical active toxoplasmic retinochoroiditis at the posterior pole or near the arcades were included in the study. Clinical diagnosis was confirmed by elevated serum levels of both toxoplasma immunoglobulins (IgG and IgM). Patients with other ocular diseases were excluded.

Patients were treated according to the standard protocol of our service, with oral sulfamethoxazole $800 \mathrm{mg} /$ trimethprim $160 \mathrm{mg}$ for 6 weeks and prednisone $0.5 \mathrm{mg} / \mathrm{kg} /$ day, tapered over 4 weeks.

All patients underwent a complete clinical examination and both SD-OCT and TC-OCT imaging at baseline and at a 6-week follow up. Patients were first imaged with TD-OCT Stratus version 3 (Carl Zeiss, Dublin, CA) and then on the SD-OCT Spectralis (Heidelberg Engineering, Heidelberg, Germany). The scan acquisition protocols for Stratus OCT included raster and radial line scan through the retinochoroiditis lesion, radial line macular scan, and a linear scan from the lesion to the fovea, whereas the acquisition protocols for Spectralis SD-OCT also included horizontal volume scans at the lesion site and at the macula. Three scans were performed on each eye, and the one with the best signal strength was considered for the final analysis.

Macular thickness was calculated by the inbuilt software analysis of the volume scans. The lesion thickness was measured by using the caliper of the SD-OCT. The measures were made from the highest point of the neurosensory retina to the retinal pigment epithelium bottom line, at the same place both at the baseline and follow up.

Two physicians with experience in interpreting OCT images independently graded both types of OCT scans. The macular scans were interpreted as normal and abnormal for both the machines. The scans were considered abnormal if they showed increased or decreased retinal thickness, cystoid changes, subretinal fluid, vitreous traction, or epiretinal membrane (ERM). Morphologic features at baseline and at 6-week follow up obtained from SD-OCT scans were compared with that obtained from TD-OCT.

\section{Statistical analysis}

Macular and lesion thickness measurements obtained by the SD-OCT were analyzed.

Data are expressed as means \pm standard error of the mean (SEM). Statistical analyses were performed using unpaired $t$-test. Pearson correlation coefficient was used to evaluate the correlation between the central foveal thickness and lesion distance from the fovea. A 95\% confidence interval and a $5 \%$ level of significance were adopted; results with a $P$-value less than or equal to 0.05 were considered significant. All statistics were calculated using Graph Pad Prism 5.0 software for Windows.

\section{Results}

Ten patients from the Retina Service of the Federal University of São Paulo were included in the study (five men and five women). The average age was 22.9 years (range from 18 to 32 years).

Toxoplasmic retinochoroiditis was diagnosed based on the clinical signs and serology. All patients presented vitritis and classic focal lesion on the posterior pole or near the vascular arcades with adjacent chorioretinal scar in $40 \%$ of them. Indeed both toxoplasma immunoglobulins were positive in all patients.

\section{Spectral domain OCT findings}

SD-OCT findings are summarized in Table 1. 
Table I Spectral domain optical coherence tomography findings

\begin{tabular}{|c|c|c|c|c|c|c|c|c|c|c|c|c|c|c|c|c|}
\hline & \multirow[t]{3}{*}{ Gender } & \multirow[t]{3}{*}{ Age } & \multicolumn{6}{|c|}{ Macula } & \multirow{3}{*}{$\begin{array}{l}\text { Dist } \\
(\mu \mathrm{m})\end{array}$} & \multicolumn{5}{|c|}{ Active Lesion } & \multirow{2}{*}{\multicolumn{2}{|c|}{$\begin{array}{l}\text { Vitreous } \\
\text { Detach- } \\
\text { ment }\end{array}$}} \\
\hline & & & \multicolumn{2}{|c|}{$\begin{array}{l}\text { Thickness } \\
(\mu \mathrm{m})\end{array}$} & \multicolumn{2}{|c|}{ Fluid } & \multicolumn{2}{|c|}{ ERM } & & \multicolumn{2}{|c|}{$\begin{array}{l}\text { Thickness } \\
(\mu \mathrm{m})\end{array}$} & \multirow[t]{2}{*}{ Scar } & \multicolumn{2}{|c|}{ ERM } & & \\
\hline & & & BL & FU & BL & FU & BL & FU & & BL & FU & & BL & FU & BL & FU \\
\hline I & $M$ & 20 & 413 & 192 & $Y$ & $\mathrm{~N}$ & $\mathrm{~N}$ & $\mathrm{~N}$ & 2225 & 431 & 184 & $Y$ & $\mathrm{~N}$ & $\mathrm{~N}$ & $\mathrm{~N}$ & $Y$ \\
\hline 2 & $\mathrm{~F}$ & 32 & 271 & 269 & $Y$ & $\mathrm{~N}$ & $Y$ & $Y$ & 5238 & 364 & 343 & $\mathrm{Y}$ & $\mathrm{Y}$ & Y & $\mathrm{Y}$ & $\mathrm{Y}$ \\
\hline 3 & $\mathrm{~F}$ & 19 & 788 & 166 & $Y$ & $\mathrm{~N}$ & $\mathrm{~N}$ & $Y$ & 4237 & 500 & 250 & $\mathrm{~N}$ & $\mathrm{~N}$ & Y & $\mathrm{N}$ & $Y$ \\
\hline 4 & $\mathrm{~F}$ & 18 & 542 & 186 & $Y$ & $Y$ & $\mathrm{~N}$ & $\mathrm{~N}$ & 681 & 571 & 215 & $\mathrm{~N}$ & $\mathrm{~N}$ & $N$ & $\mathrm{~N}$ & $Y$ \\
\hline 5 & $\mathrm{~F}$ & 18 & 183 & 186 & $\mathrm{~N}$ & $\mathrm{~N}$ & $\mathrm{~N}$ & $\mathrm{~N}$ & 3763 & 317 & 206 & $\mathrm{~N}$ & $\mathrm{~N}$ & $\mathrm{~N}$ & $Y$ & $Y$ \\
\hline 6 & M & 19 & 297 & 225 & $\mathrm{~N}$ & $\mathrm{~N}$ & $N$ & $\mathrm{~N}$ & 4876 & impos & 285 & $\mathrm{~N}$ & $Y$ & Y & $\mathrm{N}$ & $N$ \\
\hline 7 & $M$ & 26 & 237 & 228 & $\mathrm{~N}$ & $\mathrm{~N}$ & $\mathrm{~N}$ & $\mathrm{~N}$ & 6379 & 465 & 276 & $\mathrm{~N}$ & $\mathrm{~N}$ & Y & $Y$ & $Y$ \\
\hline 8 & $M$ & 28 & 236 & 232 & $N$ & $\mathrm{~N}$ & $\mathrm{~N}$ & $\mathrm{~N}$ & 6625 & 398 & 271 & $Y$ & $N$ & Y & $Y$ & $Y$ \\
\hline 9 & $\mathrm{~F}$ & 31 & 227 & 228 & $\mathrm{~N}$ & $\mathrm{~N}$ & $\mathrm{~N}$ & $\mathrm{~N}$ & 7130 & 250 & 239 & $Y$ & $N$ & $\mathrm{~N}$ & $Y$ & $Y$ \\
\hline 10 & M & 18 & 248 & 171 & $Y$ & $\mathrm{~N}$ & $N$ & $\mathrm{~N}$ & 1448 & 579 & 245 & $N$ & $\mathrm{~N}$ & $\mathrm{~N}$ & $Y$ & $Y$ \\
\hline \multicolumn{2}{|c|}{ Average } & 22.9 & 344.2 & 208.3 & & & & & 4260.2 & 430.556 & 251.4 & & & & & \\
\hline
\end{tabular}

Abbreviations: BL, baseline; Dist, distance; ERM, epiretinal membrane; F, female; FU, follow up; M, male; impos, impossible; Y, yes; N, no.

\section{Macula examination}

At baseline, the macular scans of four patients were identified as normal using SD-OCT. Macular serous detachment was observed in five patients. The presence of fluid at baseline had an indirect correlation to the distance of the retinochoroidal lesion to the fovea, but this was not statically significant $(P=0.2831)$. Retinal pigment epithelium detachment, beneath the subretinal fluid, was present in one patient (Figure 1). ERM was noticed in one patient.

The average foveal thickness decreased from $344 \pm$ $59.68 \mu \mathrm{m}$ to $208 \pm 10.40 \mu \mathrm{m}$ at the follow-up evaluation, mainly because of the subfoveal retinal detachment regression. At the 6-week follow up, the macula remained with a small retinal detachment in one patient who presented a discrete vitreous adhesion to the fovea (Figure 2).

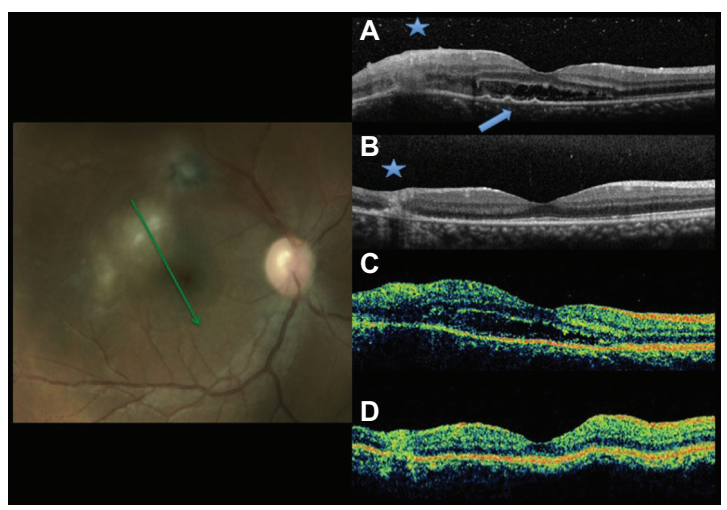

Figure I Fundus photography and radial scans at baseline by SD-OCT A) and TD-OCT C). Increased thickness and disorganization of the retinal layers at the lesion site at $A$ and $C$ (asterisk). Subretinal fluid at the foveal area. Small RPE detachments (arrowhead) only in A. Six-week follow up images by SD-OCT B) and TD-OCT D) show resolution of the subretinal fluid and decrease of the retinal thickness at the lesion site.

Abbreviations: OCT, optical coherence tomography; SD, spectral domain; $T D$, time domain.

\section{Retinochoroidal lesions}

Retinochoroidal lesions were $4260 \pm 705.2 \mu \mathrm{m}$ from the fovea (range: $681-7130 \mu \mathrm{m}$ ). Increased retinal reflectivity and thickness of the active lesion was found in all patients, using SD-OCT. Subretinal fluid near the lesion was seen in two patients (Figure 3). Retinal pigment epithelial and choriocapillaries band shadowing was noticed by both TD- and SD-OCT due to the inner retinal layers hyper-reflective at the lesion site.

At the 6-week follow up exam, seven patients showed significant decrease of the retinal thickness at the lesion site. The mean retinal thickness decrease was $179 \mu \mathrm{m}$ from the baseline $(P=0.0004)$. All patients remained with a disorganization of the retinal layers reflectivity due to scar formation. In two patients, an interruption of the inner/outer

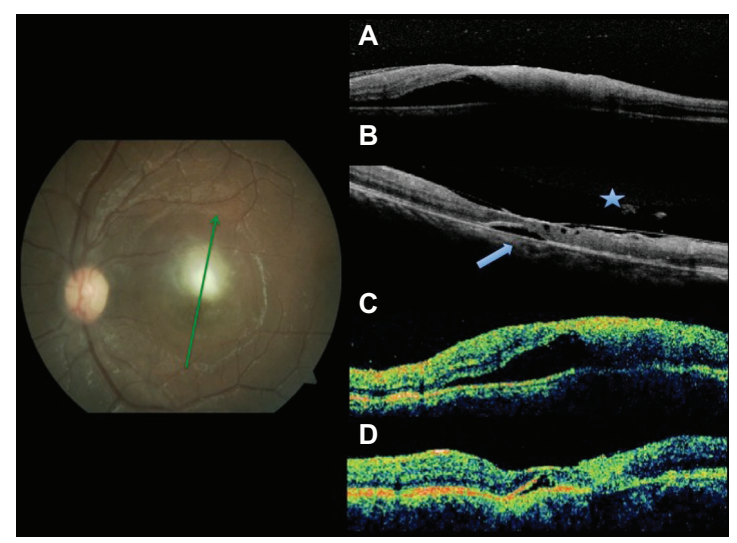

Figure 2 Fundus photography at baseline. Radial scans at baseline by SD-OCT A) and TD-OCT C) shows increased thickness at the lesion site and foveal subretinal fluid. At follow up, residual fluid (arrowhead) both by SD-OCT B) and TD-OCT D). Partial detachment of the posterior hyaloid and adhesion to the fovea only seen by SD-OCT (asterisk).

Abbreviations: OCT, optical coherence tomography; SD, spectral domain; TD, time domain. 


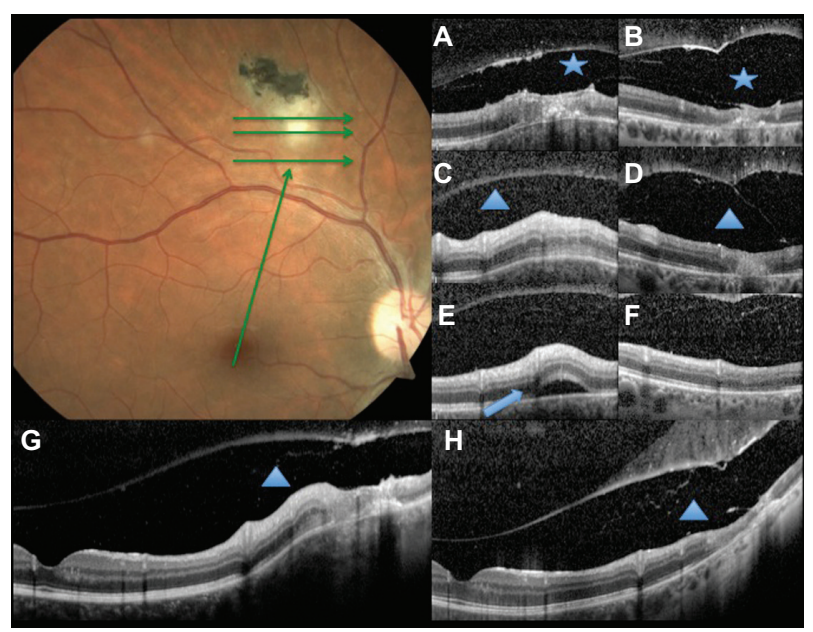

Figure 3 Baseline fundus photography. Green arrows show the scan acquisition protocol used by spectral domain optical coherence tomography. A, C, and E are from the volume scans at baseline, and $\mathrm{G}$, a linear scan from the fovea to the lesion. $\mathrm{B}, \mathrm{D}, \mathrm{F}$, and $\mathrm{H}$ correspond to the same points at follow up. Vitreoschisis (asterisk) can be noticed at baseline A) and after 6 weeks B). Note the expanded vitreous detachment at follow up. Subretinal fluid at the lesion site (arrowhead) is seen at baseline $\mathbf{E}$ ) with resolution after 6 weeks $\mathbf{F}$ ). The triangle shows the retinochoroidal lesion increased thickness at baseline $(\mathbf{C}, \mathbf{G})$ and atrophy at follow up $(\mathbf{D}, \mathbf{H})$.

segment (IS/OS) junction was noticed temporal to the fovea (Figure 4). ERM was observed near the retinochoroiditis lesion in five patients at the follow-up.

\section{Vitreous status}

We found complete posterior vitreous detachment (PVD) in one patient and partial detachment in five patients at baseline. At the follow-up, three patients presented complete PVD, two had partial detachment with adhesion to the lesion, and four patients had the hyaloid detached only over the lesion. All the

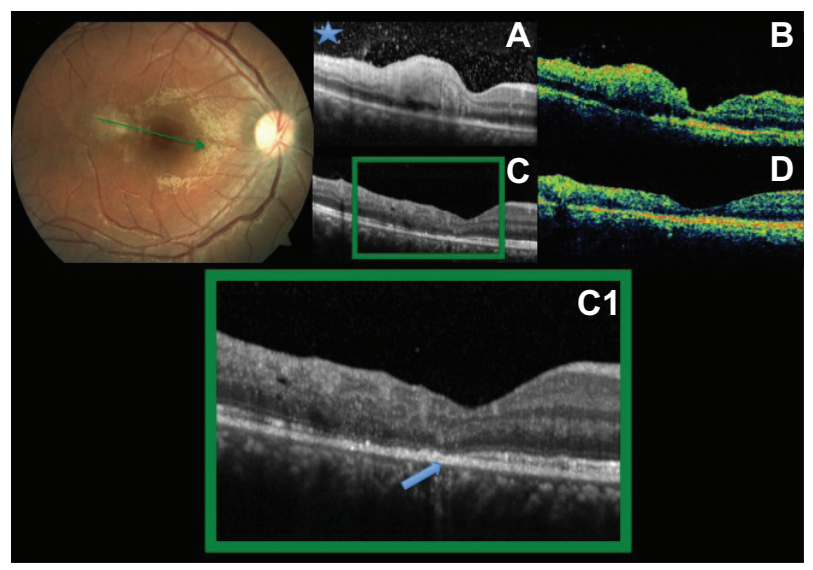

Figure 4 Baseline fundus photography. Radial scans at baseline (A, B) and after six weeks (C, D). Increased thickness at the perifoveal lesion both by SD-OCT and TDOCT. Hyper-reflective vitreous dots (asterisk) better seen in A. Decrease of the retinal thickness and disorganization of the layers are seen at follow up. Interruption of the IS/OS junction of the photoreceptor band: arrowhead C, CI.

Abbreviations: IS/OS, inner/outer segment; OCT, optical coherence tomography; $\mathrm{SD}$, spectral domain; TD, time domain. patients with vitreous detachment at the baseline showed progressive separation of the posterior hyaloid after 6 weeks.

Vitreoschisis was noticed in one patient at baseline and at the follow up (Figure 3).

Increased reflectivity of the posterior hyaloid was noticed in all patients with identifiable hyaloid at the first examination. Vitreous hyperreflective dots, probably corresponding to vitreous cells, were observed in all patients at baseline and were still seen in eight of them at follow up.

\section{Time domain OCT comparison}

Patients were imaged also with TD-OCT, and difference in the findings between SD-OCT and TD-OCT images are summarized in Table 2.

Table 2 Spectral domain versus time domain findings

\begin{tabular}{|c|c|c|c|c|}
\hline & \multicolumn{2}{|l|}{ Baseline } & \multicolumn{2}{|l|}{ Follow Up } \\
\hline & Spectralis & $\overline{\text { Stratus }}$ & Spectralis & $\overline{\text { Stratus }}$ \\
\hline \multicolumn{5}{|l|}{ Macula } \\
\hline RPE elevation & I & 0 & 0 & 0 \\
\hline Detachment & 5 & 3 & I & 1 \\
\hline Cysts & 0 & 0 & 2 & 0 \\
\hline $\begin{array}{l}\text { Epiretinal } \\
\text { membrane }\end{array}$ & I & I & 2 & 1 \\
\hline Vitreous traction & 0 & 0 & I & 0 \\
\hline Normal & 4 & 5 & 4 & 8 \\
\hline \multicolumn{5}{|l|}{ Active Lesion } \\
\hline $\begin{array}{l}\text { Increased ret } \\
\text { reflectivity }\end{array}$ & 10 & 9 & 9 & 8 \\
\hline $\begin{array}{l}\text { Disorganized } \\
\text { ret layers }\end{array}$ & 10 & 9 & 10 & 10 \\
\hline $\begin{array}{l}\text { IS/OS damage over } \\
\text { lesion }\end{array}$ & 0 & 0 & 2 & 0 \\
\hline $\begin{array}{l}\text { Epiretinal } \\
\text { membrane }\end{array}$ & 2 & 0 & 5 & 2 \\
\hline Sublesional fluid & 2 & 0 & 0 & 0 \\
\hline RPE elevation & I & 0 & I & 0 \\
\hline RPE/CC shadowing & 6 & 6 & 3 & 4 \\
\hline \multicolumn{5}{|l|}{ Vitreous status } \\
\hline Increased thickness & 9 & 6 & 3 & 3 \\
\hline Attached/ERM & 3 & 0 & I & I \\
\hline $\begin{array}{l}\text { Attached over } \\
\text { lesion }\end{array}$ & I & I & 2 & 1 \\
\hline PVD & I & 0 & 3 & 0 \\
\hline $\begin{array}{l}\text { Detached over } \\
\text { lesion }\end{array}$ & 4 & 3 & 4 & 4 \\
\hline $\begin{array}{l}\text { Expanded detach } \\
\text { on FU }\end{array}$ & 0 & 0 & 6 & 4 \\
\hline Vitreoschisis & I & 0 & 1 & 0 \\
\hline $\begin{array}{l}\text { Hyperreflective } \\
\text { vitreous dots }\end{array}$ & 10 & 9 & 8 & 4 \\
\hline $\begin{array}{l}\text { Impossible to } \\
\text { determine }\end{array}$ & I & 4 & 0 & 2 \\
\hline
\end{tabular}

Abbreviations: CC, choriocapillaris; ERM, epiretinal membrane; FU, follow up; IS/OS, inner/outer segment; PVD, posterior vitreous detachment; RPE, retinal pigment epithelium. 
The image quality was unacceptable in one patient due to the moderate vitreous opacity at baseline. Serous detachments were not identified in two patients. At follow up, intraretinal perifoveal cysts, epirretinal membranes and vitreo foveal traction could be seen by the TD-OCT.

At the active lesion, perilesional fluid and identification of damage of the IS/OS junction were not seen. The epirretinal membrane was missed by the TD-OCT in two patients at baseline and in three at follow up.

The posterior hyaloid could not be identified in four patients by TD-OCT and only in one by SD-OCT at baseline. At follow up, the vitreous status was also better analyzed by the SD-OCT. Raster and volume scans showed complete PVD and vitreoschisis, not seen by TD-OCT.

\section{Discussion}

Toxoplasmosis can cause a number of chorioretinal alterations, both at the lesion site and the macular area. Orefice et al have reported time domain OCT findings on ocular toxoplasmosis and suggest that the exam may identify morphological features not detectable on clinical examination. ${ }^{7,8}$

SD-OCT has higher axial resolution and faster acquisition speed compared with TD-OCT, which allows SD-OCT to provide not only detailed views of intraretinal microstructure, but also three-dimensional images of the macula and retinochoroiditis lesions. These enhanced views should offer physicians a better view than previous TD-OCT devices.

The current study investigated the ability to delineate and detect the macular abnormalities and the features of retinochoroiditis lesions in patients with ocular toxoplasmosis and found that SD-OCT demonstrated a significantly better image acquisition of the macular changes, such as small serous retinal detachment, epiretinal membrane, intraretinal cysts, and especially the vitreous retina interface analysis. Additionally, three-dimensional scans allow visualization of the entire scanned area, resulting in a superior ability to analyze the retinochoroiditis lesions when compared with the TD-OCT's radial line/fast macular thickness map scans.

Serous retinal detachment was a common macular abnormality found in toxoplasmic retinochoroiditis. Macular detachment was observed in $50 \%$ of the patients studied at baseline, with complete resolution in $80 \%$ of the cases after 6 weeks of treatment. The presence of residual fluid at the follow up in one patient may be associated to the proximity of the lesion to the macula, as this patient had the closest lesion-foveal distance $(681 \mu \mathrm{m})$. It can also be explained by the discrete vitreo-foveal traction found after 6 weeks in this patient.
Hystopathology findings in an eye with active toxoplasmic retinochoroiditis show cell infiltration and edema, initially in the internal retina and then secondarily involving the vitreous and the choroid. As a result of the infiltration, there is a disorganization of the retinal layers. ${ }^{9} 10$ This hystopathological feature can be correlated to the SD-OCT findings of retina hyper-reflectivity and increased thickness at the lesion. ${ }^{11}$

Lesions heal with atrophy and scarring. At the 6-week follow up, there was a significant decrease of mean retinal thickness $(P=0.0004)$. But in two patients, we still noticed increased thickness by the SD-OCT. This finding may indicate persistent activity of the lesion despite 6 weeks of treatment. Consequently, OCT may be a useful tool to indicate the lesion healing and to guide the treatment duration. ${ }^{7}$

The prevalence of posterior vitreous detachment is very low in normal subjects younger than 40 years old. However intraocular inflammation is a predisposing condition to PVD. ${ }^{12}$ Our data showed detachment of the posterior hyaloid in nine patients at follow up, compared to six at baseline. It's important to note that six patients had progression of PVD during the healing process. Those findings could only be analyzed using the volume scans of the SD-OCT.

In conclusion, SD-OCT is a useful tool in the diagnosis of macular changes related with toxoplasmic retinochoroiditis and in the management of this prevalent disease. It provides valuable information on the morphological features of the lesion, macula and vitreoretinal interface. Moreover SD-OCT had higher detection rates of macular changes and lesion features when compared with TD-OCT.

\section{Disclosure}

The authors report no conflicts of interest in this work.

\section{References}

1. Jones JL, Dargelas V, Roberts J, Press C, Remington JS, Montoya JG. Risk factors for Toxoplasma gondii infection in the United States. Clin Infect Dis. 2009;49(6):878-884.

2. Bonfioli AA, Orefice F. Toxoplasmosis. Semin Ophthalmol. 2005;20(3): 129-141.

3. Nussenblatt RB, Belfort R Jr. Ocular toxoplasmosis. An old disease revisited. JAMA. 1994;271(4):304-307.

4. Holland GN. Ocular toxoplasmosis: a global reassessment. Part II: disease manifestations and management. Am J Ophthalmol. 2004;137:1-17.

5. Gupta V, Gupta P, Singh R, Dogra MR, Gupta A. Spectral-domain Cirrus high-definition optical coherence tomography is better than time-domain Stratus optical coherence tomography for evaluation of macular pathologic features in uveitis. Am J Ophthalmol. 2008;145(6):1018-1022.

6. Wojtkowski M, Srinivasan V, Fujimoto JG, et al. Three-dimensional retinal imaging with high-speed ultrahigh-resolution optical coherence tomography. Ophthalmology. 2005;112(10):1734-1746.

7. Oréfice JL, Costa RA, Campos W, Calucci D, Scott IU, Oréfice F. Third-generation optical coherence tomography findings in punctate retinal toxoplasmosis. Am J Ophthalmol. 2006;142(3):503-505. 
8. Oréfice JL, Costa RA, Oréfice F, Campos W, da Costa-Lima D Jr, Scott IU. Vitreoretinal morphology in active ocular toxoplasmosis: a prospective study by optical coherence tomography. Br J Ophthalmol. 2007;91(6):773-780.

9. Frenkel J, Jacobs I. Ocular toxoplasmosis: pathology, diagnosis and treatment. Arch Opthalmol. 1958;59:260.

10. Gallagher MJ, Yilmaz T, Cervantes-Castañeda RA, Foster CS. The characteristic features of optical coherence tomography in posterior uveitis. Br J Ophthalmol. 2007;91(12):1680-1685.
11. Garg S, Mets MB, Bearelly S, Mets R. Imaging of congenital toxoplasmosis macular scars with optical coherence tomography. Retina. 2009;29(5):631-637.

12. Bosch-Driessen LE, Berendschot TT, Ongkosuwito JV, Rothova A. Ocular toxoplasmosis: clinical features and prognosis of 154 patients. Ophthalmology. 2002;109(5):869-878.

\section{Publish your work in this journal}

Clinical Ophthalmology is an international, peer-reviewed journal covering all subspecialties within ophthalmology. Key topics include: Optometry; Visual science; Pharmacology and drug therapy in eye diseases; Basic Sciences; Primary and Secondary eye care; Patient Safety and Quality of Care Improvements. This journal is indexed on

\section{Dovepress}

PubMed Central and CAS, and is the official journal of The Society of Clinical Ophthalmology (SCO). The manuscript management system is completely online and includes a very quick and fair peer-review system, which is all easy to use. Visit http://www.dovepress.com/ testimonials.php to read real quotes from published authors. 